01

\title{
Зависимость энергетического распределения распыленных атомов от закона межатомного взаимодействия
}

\author{
(С) А.И. Толмачев, ${ }^{1}$ Л. Форлано ${ }^{2}$ \\ ${ }^{1}$ Российский новый университет, \\ 105005 Москва, Россия \\ ${ }^{2}$ Universita della Calabria, \\ 87036 Cosenza, Italia \\ e-mail: tolmachev.alex@rambler.ru, forlano@vegachess.com
}

Поступило в Редакцию 11 октября 2019 г.

В окончательной редакции 11 октября 2019 г.

Принято к публикации 9 января 2019 г.

\begin{abstract}
Проведен расчет энергетического распределения атомов, распыленных при бомбардировке твердого тела ионами. Теоретическое рассмотрение основано на решении системы двух уравнений переноса методом дискретных потоков. Показано, что при малых энергиях распыленных атомов их энергетическое распределение описывается универсальной формулой, содержащей единственную постоянную. Значение постоянной увеличивается с ростом массы ионов и уменьшается при переходе от резерфордовского взаимодействия к взаимодействию по закону твердых сфер.
\end{abstract}

Ключевые слова: ионная бомбардировка, распыление, теоретический анализ, сечение рассеяния, энергетическое распределение.

DOI: 10.21883/JTF.2020.06.49271.335-19

\section{Введение}

Теоретическое исследование явления распыления твердых тел под действием ионной бомбардировки имеет большое значение для получения информации о поверхностных слоях вещества, а также для интерпретации многочисленных экспериментальных данных $[1,2]$. Большинство экспериментов относится к энергетической зависимости коэффициента распыления для различных комбинаций ионов и атомов мишени. Величина коэффициента распыления зависит главным образом от числа атомов с малыми энергиями.

Энергетическое распределение распыленных атомов, проинтегрированное по всем углам вылета, при малых энергиях имеет вид

$$
Y(u) d u=C\left(\ln \frac{1}{u}\right)^{-3 / 2} \frac{d u}{u^{2}}, \quad u=\frac{E}{E_{0}} \ll 1,
$$

где $u$ - относительная энергия, $E_{0}$ и $E-$ энергии ионов и атомов соответственно. Обратный квадратичный член в (1) является результатом решения задачи в приближении бесконечной мишени, использованного в теории распыления Зигмунда [3]. Дополнительный логарифмический множитель появляется после учета поверхности мишени. Коэффициент $C$ зависит от отношения масс иона и атома $A=M_{1} / M_{2}$ а также от сечения рассеяния, связанного с законом межатомного взаимодействия. Приближенные значения $C$ были найдены в [4] методом сферических функций, и в $[5,6]$ - методом дискретных потоков. В настоящей работе получены точные зависимости коэффициента $C$ от отношения $A$ для различных сечений рассеяния - от резерфордовского до сечения, соответствующего потенциалу твердых сфер. Рассматривается случай легких ионов, при котором масса иона не превышает массы атома мишени, $A \leq 1$.

\section{Сечение рассеяния}

В работах $[3,4,7]$ используется сечение рассеяния в виде степенной функции от энергии $T$, переданной при столкновении

$$
d \sigma(E, T)=C_{m} E^{m} T^{-1-m} d T .
$$

Значение параметра $m=+1$ соответствует взаимодействию по закону твердых сфер, $m=-1$ - резерфордовскому взаимодействию. Однако при $m \geq 0$ соотношение (2) приводит к бесконечному полному сечению. Это затрудняет теоретическое рассмотрение и, кроме того, делает невозможным проверку результатов с помощью программ компьютерного моделирования, основанных на модели бинарных столкновений.

В настоящей работе мы используем экранированное сечение рассеяния [8-10], для которого полное сечение всегда конечно:

$$
d \sigma(E, T)=\frac{\gamma \eta(1+\eta) d T}{E(\gamma \eta+T / E)^{2}}, \quad \text { где } \quad \eta=\frac{1}{4 \varepsilon(1+\varepsilon)}
$$

- параметр экранирования, $\varepsilon$ - приведенная энергия, $\gamma=4 A /(1+A)^{2}[10]$. Предельный случай $\varepsilon=0$ описывает рассеяние на потенциале твердых сфер, а предельный случай $\varepsilon \gg 1$ - резерфордовское рассеяние на кулоновском потенциале. Другим типам атомных потенциалов 
соответствуют промежуточные значения приведенной энергии.

Переход в (3) к углу рассеяния налетающей частицы в лабораторной системе координат $\Omega$ дает сечение рассеяния $d \sigma(A, \Omega)$ для столкновений ионов и атомов мишени и сечение рассеяния $d \sigma(1, \Omega)$ для столкновений каскадных атомов между собой. Соответствующие отношения $\Delta_{1}$ и $\Delta_{2}=1-\Delta_{1}$ энергии частицы после столкновения к ее энергии до столкновения могут быть найдены из кинематических уравнений.

\section{Уравнения переноса}

В каскадном размножении участвуют два сорта частиц - рассеянные ионы и атомы отдачи, выбитые из положений равновесия после столкновений с ионами и ранее появившимися атомами отдачи, поэтому теоретический анализ включает в себя решение системы двух уравнений переноса для функций распределения рассеянных ионов $f(x, \mu, u)$ и каскадных атомов $g(x, \mu, u)$. Функции распределения зависят от нормированной глубины мишени $x$, косинуса $\mu$ угла между скоростью частицы и внутренней нормалью к поверхности мишени, a также от относительной энергии $u$. Функция распределения рассеянных ионов, полученная из решения первого уравнения, играет роль функции источника атомов отдачи во втором уравнении.

Граничное условие для функции распределения рассеянных ионов указывает на то, что они падают по нормали к поверхности мишени и их начальная энергия равна $E_{0}: f(0, \mu, u)=\delta(1-\mu) \delta(1-u)$ для $\mu>0$. Граничное условие для функции распределения каскадных атомов показывает, что при входе в мишень каскадные атомы отсутствуют: $g(0, \mu, u)=0$ для $\mu>0$.

Выполним преобразование Меллина по энергетической переменной

$$
\begin{aligned}
& f_{s}(x, \mu)=\int_{0}^{1} u^{s} f(x, \mu, u) d u, \\
& g_{s}(x, \mu)=\int_{0}^{1} u^{s} g(x, \mu, u) d u,
\end{aligned}
$$

и запишем систему уравнений переноса для ионов и атомов

$$
\begin{gathered}
\mu \frac{\partial f_{s}}{\partial x}+f_{s}=\int_{-1}^{1} p_{1}\left(A, \mu, \mu^{\prime}\right) f_{s}\left(x, \mu^{\prime}\right) d \mu^{\prime} \\
\mu \frac{\partial g_{s}}{\partial x}+g_{s}=\int_{-1}^{1}\left[p_{1}\left(1, \mu, \mu^{\prime}\right)+p_{2}\left(1, \mu, \mu^{\prime}\right)\right] g_{s}\left(x, \mu^{\prime}\right) d \mu^{\prime} \\
+Q(x, \mu)
\end{gathered}
$$

где функция источника имеет вид

$$
Q(x, \mu)=\int_{-1}^{1} p_{2}\left(A, \mu, \mu^{\prime}\right) f_{s}\left(x, \mu^{\prime}\right) d \mu^{\prime}
$$

Уравнение (6) описывает рассеяние ионов в полубесконечной мишени. Это уравнение было подробно исследовано Чандрасекаром [11] для различных сечений рассеяния. В результате решения мы получаем значения функции $f_{s}(x, \mu)$ для отрицательных $\mu$, и после интегрирования по углам вылета - величину коэффициента отражения как функцию параметра $s$ :

$$
R_{N}(s)=\int_{-1}^{0}|\mu| f_{s}(0, \mu) d \mu .
$$

Уравнение (7) описывает каскадное размножение атомов отдачи. Интегрирование решения $g_{s}(x, \mu)$ по углам вылета дает коэффициент распыления

$$
Y_{N}(s)=\int_{-1}^{0}|\mu| g_{s}(0, \mu) d \mu .
$$

Угол рассеяния в лабораторной системе координат $\Omega$ связан с направляющими косинусами соотношением

$$
\cos \Omega=\mu \mu^{\prime}+\sqrt{1-\mu^{2}} \sqrt{1-\mu^{\prime 2}} \cos \varphi,
$$

где $\varphi$ - азимутальный угол. В [11] решалась задача о переносе света в межзвездной атмосфере. Это соответствует случаю $A=0$ отсутствию передачи энергии и простому виду сечения рассеяния в виде линейной комбинации первых многочленов Лежандра $P_{n}(\cos \Omega)$, $n=0,1,2$. В случае отражения и распыления частиц мы имеем $A>0$ и должны учитывать как передачу энергии, так и сложный вид сечения рассеяния, поэтому угловые функции в уравнениях (6)-(8) определялись численно, путем усреднения по азимутальному углу сечений рассеяния, взятых с весовыми функциями, отвечающими за передачу энергии:

$$
\begin{aligned}
& p_{1}\left(A, \mu, \mu^{\prime}\right)=\frac{1}{\pi} \int_{0}^{\pi} \Delta_{1}^{s}(A, \Omega) \sigma(A, \Omega) d \varphi, \\
& p_{2}\left(A, \mu, \mu^{\prime}\right)=\frac{1}{\pi} \int_{0}^{\pi} \Delta_{2}^{s}(A, \Omega) \sigma(A, \Omega) d \varphi .
\end{aligned}
$$

Необходимо подчеркнуть, что определение энергетического распределения при малых энергиях не требует выполнения обратного преобразования Меллина, а только исследования решения в окрестности точки $s \approx 1$. 


\section{Метод решения}

Для решения системы уравнений (6)-(8) мы использовали метод дискретных потоков [12]. Интервал интегрирования по $\mu^{\prime}$ разбивался на $N$ равных частей, и рассматривались значения неизвестных функций распределения в $N$ точках, после чего интегродифференциальные уравнения сводились к системам $N$ линейных дифференциальных уравнений первого порядка. Решение разлагалось в ряд по собственным функциям $F_{n}(\mu)$ и $G_{n}(\mu)$ :

$$
\begin{gathered}
f_{s}(x, \mu)=\sum_{n=1}^{N} a_{n} F_{n}(\mu) \exp \left(-\lambda_{n} x\right), \\
g_{s}(x, \mu)=\sum_{n=1}^{N} b_{n} G_{n}(\mu) \exp \left(-\Lambda_{n} x\right)+G(x, \mu),
\end{gathered}
$$

где

$$
G(x, \mu)=\sum_{n=1}^{N} c_{n} F_{n}(\mu) \exp \left(-\lambda_{n} x\right) .
$$

Собственные значения и собственные функции определялись из решения интегральных уравнений

$$
\begin{aligned}
\left(1-\lambda_{n} \mu\right) F_{n}(\mu)= & \int_{-1}^{1} p_{1}\left(A, \mu, \mu^{\prime}\right) F_{n}\left(\mu^{\prime}\right) d \mu^{\prime}, \\
\left(1-\Lambda_{n} \mu\right) G_{n}(\mu)= & \int_{-1}^{1}\left[p_{1}\left(1, \mu, \mu^{\prime}\right)+p_{2}\left(1, \mu, \mu^{\prime}\right)\right] \\
& \times G_{n}\left(\mu^{\prime}\right) d \mu^{\prime},
\end{aligned}
$$

которые после применения метода дискретных потоков сводились к системам линейных алгебраических уравнений. Собственные значения квадратной матрицы размером $N \times N$ находились методом вращений. Коэффициенты разложений (14)-(16) получались из граничных условий. Значение коэффициента $C$ в энергетическом распределении (1) определялось как предел

$$
C=\lim _{s \rightarrow 1} \frac{1-R_{N}(s)-Y_{N}(s)}{2 \sqrt{\pi(s-1)}} .
$$

Сходимость решения контролировалась путем увеличения числа дискретных потоков вплоть до максимального значения $N=500$. Корректность метода была проверена на тестовых задачах с известными аналитическими решениями [11], а также с помощью программы компьютерного моделирования PAOLA [10].

\section{Результаты}

Рис. 1 показывает зависимость коэффициента $C$ в уравнении (1) от отношения масс $A$ и вида межатомного

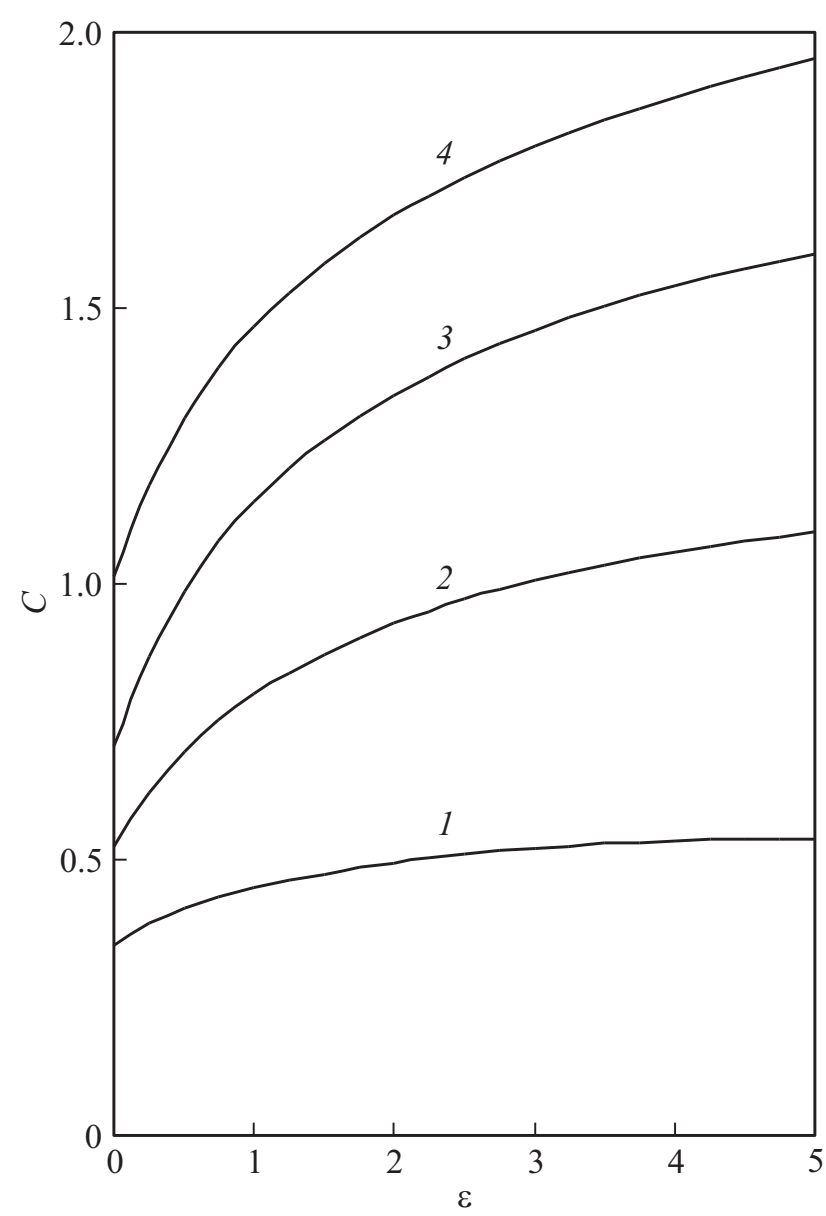

Рис. 1. Изменение коэффициента $C$ при переходе от взаимодействия по закону твердых сфер (малые $\varepsilon$ ) к резерфордовскому взаимодействию (большие $\varepsilon$ ). Отношение масс: $A=0.0001$ (1), 0.1 (2), 0.5 (3), 1 (4).

взаимодействия. Из рисунка следует, что число низкоэнергетических распыленных атомов увеличивается с ростом $A$ а также при постепенном переходе от взаимодействия по закону твердых сфер к резерфордовскому взаимодействию. При больших значениях приведенной энергии, соответствующих резерфордовскому взаимодействию, коэффициент $C$ стремится к постоянному значению, зависящему только от отношения масс. Любопытно отметить, что при малых массах ионов $A \ll 1$ коэффициент $C$ стремится к конечному значению, а не к нулю, как этого можно было бы ожидать по аналогии с соответствующим коэффициентом для энергетического распределения отраженных ионов [13].

На рис. 2 изображено изменение коэффициента $C$ при учете неупругих потерь энергии. Для качественной оценки мы использовали простую модель, при которой неупругие потери являются локальными и пропорциональными энергии. Это означает, что в каждом упругом столкновении частицы энергии $E$ теряется дополнительная энергия, равная $D E$, где $D<1-$ безразмерный параметр. Если неупругие потери отсутствуют, то $D=0$. 


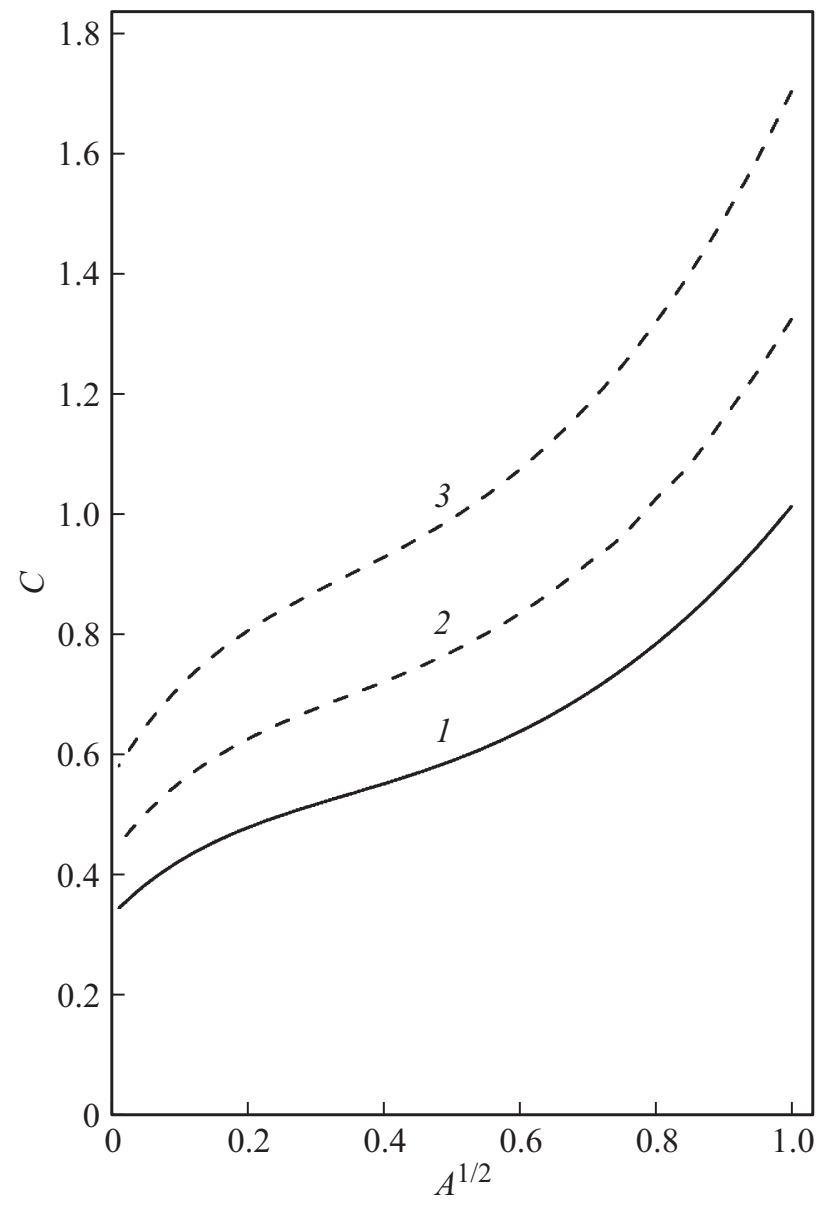

Рис. 2. Взаимодействие по закону твердых сфер. Зависимость коэффициента $C$ от отношения масс $A$ и коэффициента неупругих потерь энергии: $D=0$ (неупругие потери отсутствуют, (кривая 1)) 0.3 (2), 0.6 (3).

Как и следовало ожидать, неупругие потери энергии увеличивают число распыленных атомов с малыми энергиями.

\section{Заключение}

Показано, что экранированное сечение рассеяния (3) является разумной альтернативой широко используемому в теории распыления степенному сечению (2). Конечность полного сечения дает возможность сформулировать систему уравнений переноса в явном виде и получить энергетические распределения распыленных атомов. Интегрирование найденных распределений по энергиям в пределах от высоты потенциального барьера до пороговой энергии может дать хорошую оценку значений коэффициента распыления для различных энергий ионов и комбинаций ион-мишень. Продемонстрирована эффективность метода дискретных потоков для численного решения уравнений переноса.

\section{Конфликт интересов}

Авторы заявляют, что у них нет конфликта интересов.

\section{Список литературы}

[1] Борисов А.М., Машкова Е.С. Физические основы ионнолучевых технологий. II. Распыление поверхности твердых тел. М.: МАКС Пресс, 2013. 196 с.

[2] Behrish R., Eckstein W. (editors). Sputtering by Particle Bombardment. Berlin, Springer, 2007. 470 p.

[3] Sigmund P. // Phys. Rev. 1969. Vol. 184. N 2. P. 383-416.

[4] Waldeer K.T., Urbassek H.M. // Appl. Phys. 1988. Vol. A 45. N 3. P. 207-215.

[5] Tolmachev A.I. // Nucl. Instr. Methods. 1994. Vol. B 93. N 4. P. 415-420.

[6] Барат А.А., Манухин В.В. // ЖТФ. 2009. Т. 79. Вып. 2. C. 117-124. [Barat A.A., Manukhin V.V. // Tech. Phys. 2009. Vol. 54. N 2. P. 281-288.]

[7] Roosendaal H.E., Sanders J.B. // Radiation Effects. 1980. Vol. 52. P. 137-144.

[8] Leibfried G., Oen O.S. // J. Appl. Phys. 1962. Vol. 33. N 7. P. 2257-2262.

[9] Eckstein W. Computer Simulation of Ion-Solid Interactions. Berlin, Springer, 1991. Chapter 4.

[10] Толмачев А.И., Форлано Л. // ЖТФ. 2018. Т. 88. Вып. 10. C. 1502-1505. [Tolmachev A.I., Forlano L. // Tech. Phys. 2018. Vol. 63. N 10. P. 1455-1458.]

[11] Chandrasekhar S. Radiative Transfer. Oxford, Clarendon Press, 1950. 393 p.

[12] Толмачев А.И. // Известия АН СССР. Серия физическая. 1990. T. 54. Вып. 7. С. 1247-1249. [Tolmachev A.I. // Bulletin of the Academy of Sciences of the USSR. Physical Series. 1990. Vol. 54. N 7. P. 13-15.]

[13] Толмачев А.И., Форлано Л. // Поверхность. 2020. Вып. 5. C. 89-92. 